\title{
Reconstruction of Serially Acquired Slices Using Physics-Based Modeling
}

\author{
Stelios Krinidis, Chistophoros Nikou, Member, IEEE, and Ioannis Pitas, Senior Member, IEEE
}

\begin{abstract}
This paper presents an accurate, computationally efficient, fast, and fully automated algorithm for the alignment of two-dimensional (2-D) serially acquired sections forming a 3-D volume. The approach relies on the determination of interslice correspondences. The features used for correspondence are extracted by a 2-D physics-based deformable model parameterizing the object shape. Correspondence affinities and global constrains render the method efficient and reliable. The method accounts for one of the major shortcomings of 2-D slices alignment of a 3-D volume, namely variable and nonuniform thickness of the slices. Moreover, no particular alignment direction is privileged, avoiding global offsets, biases, and error propagation. The method was evaluated on real images and the experimental results demonstrated its accuracy, as reconstruction errors were smaller than 1 degree in rotation and smaller than 1 pixel in translation.
\end{abstract}

Index Terms-Image registration, misalignment, physics-based deformable modeling, registration error, 2-D serially acquired images.

\section{INTRODUCTION}

$\mathbf{I}$ $\mathrm{N}$ the past few years, the three-dimensional (3-D) medical image reconstruction (e.g., CT, tissue sections, and autoradiographic slices) has been a very important topic in biomedical research. As one can very easily establish, a variety of imaging devices has been used in 3-D medical imaging. Various 3-D image representations have been introduced, the dominant one being the representation of 3-D images as sequences of 2-D parallel image slices. The 2-D slices may be aligned or not, depending on the application. To name a few examples, MRI images are aligned, whereas biological tissue image slices obtained using a microtome and a microscope are not. In fact, all slices obtained though physical sectioning are not aligned. One can safely deduce from the above that the reconstruction of the corresponding 3-D volumes of the aforementioned data sets, performed by the registration of the 2-D slices, has become increasingly valuable. Moreover, multiple slice registration is essential in order to visualize these structures in 3-D space and perform morphometric analysis (i.e., surface/volume representation). Past research has produced a number of alignment algorithms that are related to the registration of 2-D slices. A review of general medical image registration methods is presented in [1]-[3].

The 3-D reconstruction methods (from 2-D parallel images) may be classified in the following categories: fiducial marker-

\footnotetext{
Manuscript received January 17, 2003; revised September 1, 2003 and September 5, 2003.

The authors are with the Department of Informatics, Aristotle University of Thessaloniki, 54124 Thessaloniki, Greece (e-mail: pitas@zeus.csd.auth.gr). Digital Object Identifier 10.1109/TITB.2003.821335
}

based methods [4], feature-based methods using contours [5], methods using crest lines or characteristic points extracted from the images [6], [7], and gray level-based registration techniques using the intensities of the entire image [8]-[11]. Most of the aforementioned techniques do not consider one of the major difficulties involved in medical image registration, i.e., the slice variation and non uniformity of thickness. To be more precise, it is often the case that slices are obtained using methods (such as physical sectioning) that prevent them from being always equally thick. This can happen not only when the acquired slices are parallel to each other, but also when the slices themselves may exhibit non uniformity in thickness, which is the most interest to us. Moreover, these effects are more pronounced when distant slices are involved in the registration. From this point of view, a registration method must be robust to missing data or outliers [8], [11]. Furthermore, sequential slice registration (i.e., registering the second slice with respect to the first one, the third with respect to the second, etc.) leads to a different type of misregistration. In this case, if an error occurs in the registration of a slice with respect to the preceding slice, this error will propagate through the entire volume. If the number of slices to be registered is large, a global offset of the volume may be observed, due to error accumulation [9].

In this paper, a solution to the above mentioned shortcomings is presented. A method determining correspondences between serially acquired slices is proposed. The features used for correspondence are extracted by a finite element-based model parameterizing the object shape. Although, finite element methods (FEMs) are a powerful tool in computer vision applications, they have not yet been extensively considered for the registration of serially acquired slices.

Our method works as follows. We model each slice contour by a set of contour nodes using a 2-D physics-based deformable contour modeling technique [12], [13]. We establish mode correspondences among contour nodes of consecutive slices using special mode affinity metrics. We automatically prune node correspondences that do not make sense. Finally, we align the various slices by using valid node correspondences and the calculation of the optimal rotation and translation matrices.

Our approach was motivated by a FEM technique presented in [14], which consists in determining correspondences between objects for recognition using eigen-decomposition analysis. However, our method determines correspondences between slices exploiting contour information obtained by the free vibrations of an initial circular model (physics-based modeling) [12], [13]. Model matching using physics-based models renders our method robust to missing data. Furthermore, global affinities between correspondences and their filtering render 
our method more efficient and reliable, overcoming major alignment problems such as nonuniformity in slices thickness.

The remainder of the paper is organized as follows. The physics-based deformable model used as the feature generator is presented in Section II. In Section III, the determination of the correspondences between slices is introduced. Experimental results are presented in Section IV and conclusions are drawn in Section V.

\section{2-D Physics-BASED DEFORMABLE CONTOUR MODELING}

In this section, we introduce our physically based deformable model, which we use for parameterizing the object shape. We consider both the surface and volume properties of the objects at hand. We restrict ourselves to elastic deformations, i.e., we assume that the object recovers its original configuration as soon as all applied forces causing the deformation are removed.

Each slice is separately parameterized by an elastic physicsbased deformable model [12], [13]. The model consists of $N$ virtual masses sampled on a circular structure, following a circle that surrounds the 2-D object slice as shown in Fig. 1(a). Each model node has a mass $m$ and is connected to its two neighbors with identical springs of stiffness $k$. Constants $k$ and $m$ describe the physical characteristics of the model and determine its physical behavior. When $k$ increases, the object tends to behave as a rigid one, which means, in practice, that the model can be spatially moved without any deformation. On the other hand, when $m$ increases, the model tends to be treated as a fully deformable one, which means that each force affects only the node (mass) it is applied to. Furthermore, these model nodes are points on the object contour at equilibrium and do not represent interior object regions. The node coordinates of the model under examination are stacked in vector

$$
\mathbf{v}_{t}=\left[x_{1}, y_{1}, \ldots, x_{N}, y_{N}\right]^{T}
$$

where $N$ is the number of nodes (masses) of the model and $t$ denotes the $t$ th deformation time instance. In the following, $v_{t}^{i}$ denotes the $i$ th component of vector $\mathbf{v}_{t}$. The model under study, is a physics-based system governed by the fundamental dynamics equation

$$
f_{e}\left(v_{t}^{i}\right)+f_{d}\left(v_{t}^{i}\right)+f_{e x t}\left(v_{t}^{i}\right)=m_{i} \ddot{v}_{t}^{i}, \quad i=1,2, \ldots, N
$$

where $m_{i}$ is the mass of the point under study and $\ddot{v}_{t}^{i}$ its acceleration under total load of forces ( $i$ th component of vector $\ddot{\mathbf{v}}_{t}$ ). $f_{d}(\cdot)$ is a damping force, $f_{\text {ext }}(\cdot)$ is the external load on node under study, and $f_{e}(\cdot)$ is the elastic force due to the neighbors of each node. The above governing equation is expressed for all model nodes, leading to a nonlinear system of coupled differential equations, since the displacement of a node depends on the displacement of its neighbors that affect the term $f_{e}(\cdot)$.

In order to solve this system of equations, we propose to set the natural length of the springs to zero. The length of the springs is included in the elastic force $f_{e}(\cdot)$ equation. This assumption does not import any restriction to the initial configuration of the model, if we add an equilibrium force $f_{e q}\left(v_{t}^{i}\right)=-f_{e}\left(v_{t}^{i}\right)$ in (2). This force keeps the model inflated so that it does not

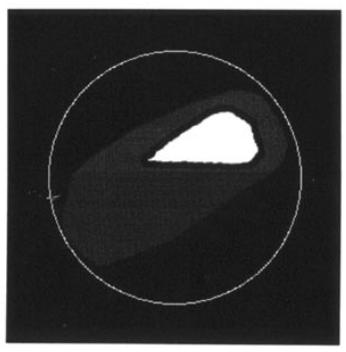

(a)

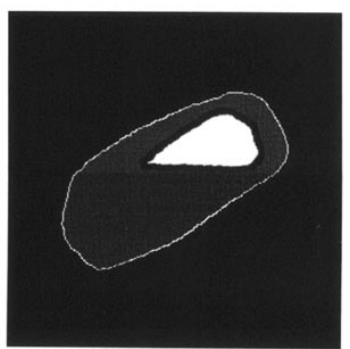

(b)
Fig. 1. Contour modeling of a 2-D slice of a 3-D tooth germ volume. (a) The initial circular model initialized around the object to be parameterized. (b) The deformable model at equilibrium ( $25 \%$ of the vibration modes are kept).

shrink to a point. We assume that, at any future time, this equilibrium force is constant. Hence, the natural state of the model is its initial configuration. This assumption has a main advantage, that the model can be considered within the framework of linear elasticity, i.e., (2) is transformed in a set of linear differential equations with node displacements decoupled in each coordinate, regardless of the magnitude of the displacements. To enforce the assumption of constant equilibrium force $f_{e q}(\cdot)$, the angular variations of the spring orientation should be sufficient small $\left(<15^{\circ}\right)$, in which case the aforementioned approximation is valid [15], [16].

The governing equation can now be written in a matrix form [17]

$$
\mathbf{M} \ddot{\mathbf{u}}+\mathbf{C} \dot{\mathbf{u}}+\mathbf{K u}=\mathbf{f}_{t}
$$

where $\mathbf{u}$ is the nodal displacements vector $\left(\mathbf{u}=\mathbf{v}_{t}-\mathbf{v}_{t_{0}}\right) . \mathbf{M}$, $\mathbf{C}$, and $\mathbf{K}$ [12], [13], [17], [18] are the mass, damping, and stiffness matrices of the model, respectively, and $\mathbf{f}_{t}$ is the external force vector, usually resulting from the attraction of the model by the object contour (usually based on the Euclidean distance between the object contour and the node coordinates [19], [20]). Equation (3) is a finite element formulation of the deformation process.

Instead of finding directly the equilibrium solution of (3), one can transform it by a basis change

$$
\mathbf{u}=\Psi \tilde{\mathbf{u}}
$$

where $\boldsymbol{\Psi}$ is the square nonsingular transformation matrix of order $N$ to be determined and $\tilde{\mathbf{u}}$ is referred to as the generalized displacement vector. One effective way of choosing $\Psi$ is setting it equal to matrix $\boldsymbol{\Phi}$, whose entries are the eigenvectors of the generalized eigenproblem

$$
\begin{aligned}
\mathbf{K} \boldsymbol{\phi}_{i} & =\omega_{i}^{2} \mathbf{M} \boldsymbol{\phi}_{i} \\
\mathbf{u} & =\boldsymbol{\Phi} \tilde{\mathbf{u}}=\sum_{i=1}^{N} \tilde{u}_{i} \boldsymbol{\phi}_{i} .
\end{aligned}
$$

Equation (6) is referred to as the modal superposition equation. The $i$ th eigenvector, i.e., the $i$ th column of $\Phi$, denoted by $\phi_{i}$, is also called the $i$ th vibration mode, $\tilde{u}_{i}$ (the $i$ th scalar component of $\tilde{\mathbf{u}})$ is its amplitude, and $\omega_{i}$ is the corresponding eigenvalue (also called frequency). If the matrix $\tilde{\mathbf{C}}=\boldsymbol{\Phi}^{T} \mathbf{C} \boldsymbol{\Phi}$ is diagonal (standard Rayleigh hypothesis [12]), then, in the modal space, 
the governing matrix-form equations are decoupled into $N$ scalar equations

$$
\ddot{\tilde{u}}_{t}^{i}+\tilde{c}_{i} \dot{\tilde{u}}_{t}^{i}+\omega_{i}^{2} \tilde{u}_{t}^{i}=\tilde{f}_{t}^{i}, \quad i=1, \ldots, N .
$$

Solving these equations at time $t$ leads to $\tilde{u}_{t}^{i}$, and the displacement $\tilde{\mathbf{u}}$ of the model nodes is obtained by the modal superposition (6).

In practice, we wish to approximate nodal displacements $\mathbf{u}$ by $\hat{\mathbf{u}}$, which is the truncated sum of the $N^{\prime}$ low-frequency vibration modes, where $N^{\prime} \ll N$

$$
\mathbf{u} \approx \hat{\mathbf{u}}=\sum_{i=1}^{N^{\prime}} \tilde{u}_{i} \phi_{i}
$$

Eigenvectors $\phi_{i}$ form the reduced modal basis of the system. This is the major advantage of modal analysis: it is solved in a subspace corresponding to the $N^{\prime}$ truncated low-frequency vibration modes of the deformable structure [12], [13], [15]. The number of vibration modes $N^{\prime}$ retained in the object description is chosen so as to obtain a compact but adequately accurate contour representation. A typical a priori value for $N^{\prime}$, covering many types of standard deformations is equal to one-quarter of the number of the vibration modes. For instance, if we consider a model of 1000 nodes and a volume of 128 slices, an equation system (3) comprising of $2 \times 1,000 \times 128=256000$ equations has to be solved. It is clear that the equation system in the reduced subspace (25\% of the vibration modes were kept) would noticeably reduce the computation time, as it would contain $2 \times 1000 \times 128 \times 25 \%=64000$ equations. Besides reducing the deformation execution time, deformation in a reduced modal space is directly related to the volume slices thickness nonuniformity problem and to the erroneous contour cuts created by tissue tears. These contour jaggy detail information lies in the high-frequency modes. Thus, by removing these frequencies and taking into account only a sufficient number of low-frequency vibration modes, we solve the contour jaggy problems (thickness problem and erroneous contour cuts).

An important advantage of the formulations described so far, in the full as well as the truncated modal space, is that the vibration modes (eigenvectors) $\phi_{i}$ and the frequencies (eigenvalues) $\omega_{i}$ of a closed circular topology have an explicit formulation [12] and they do not have to be computed using eigen-decomposition techniques

$$
\begin{aligned}
\omega_{i}^{2} & =\frac{4 k}{m} \sin ^{2}\left(\frac{\pi i}{N}\right) \\
\boldsymbol{\phi}_{i} & =\left[\ldots, \cos \frac{2 \pi i j}{N}, \ldots\right]^{T}
\end{aligned}
$$

where $i \in\{1,2, \ldots, N\}$ and $j \in \mathcal{B}(N) . \mathcal{B}(N)$ is the first Brillouin zone [12] and is equal to $\{-(N / 2)+1, \ldots, N / 2\}$ for $N$ even, and $\{-((N-1) / 2), \ldots,((N-1) / 2)\}$ for $N$ odd. This is the reason we have chosen and used a circular model topology, in spite of its low efficiency in describing complex shapes (e.g., objects with more that one contours and/or large contour curvature).

In many computer vision applications [13], when the initial and the final deformable contour states are known, it is assumed that a constant force load $\mathbf{f}$ is applied to the contour model. Thus, (3) is called the equilibrium governing equation and corresponds to the static problem

$$
\mathbf{K u}=\mathbf{f}
$$

In the new basis, (11) is simplified to $2 N$ scalar equations:

$$
\omega_{i}^{2} \tilde{u}_{i}=\tilde{f}_{i} .
$$

In (12), $\omega_{i}$ designates the $i$ th eigenvalue. The scalar $\tilde{u}_{i}$ is the amplitude of the corresponding vibration mode (corresponding to eigenvector $\phi_{i}$ ). Equation (12), indicates that, instead of computing the displacements vector $\mathbf{u}$ from (11), we can compute its decomposition in terms of the vibration modes of the original circular model.

Thus, the deformations of the described deformable model can be given by

$$
u_{i}^{\prime}=\sum_{j}^{N} \frac{\sum_{n=1}^{N}\left[f_{n} \phi_{n}(j)\right]}{\left(1+\omega_{j}^{2}\right) \sum_{n=1}^{N} \phi_{n}^{2}(j)} \phi_{i}(j)
$$

and the final contour representation $\mathbf{v}$ is finally given by adding the deformations to the initial circular model $\mathbf{v}_{0}$

$$
\mathbf{v}=\mathbf{v}_{0}+\mathbf{u}^{\prime} .
$$

In our case, each slice of the 3-D volume is described in terms of vibrations of an initial model. Figs. 1 and 2 illustrate the vibration modes based parameterization of the 2-D slices of a tooth germ volume. The $25 \%$ lowest frequency modes were retained for this representation as this truncated description provides a satisfactory compromise between accuracy and complexity of the representation. The circular contour model is initialized around each slice [Fig. 1(a)] and the vibration amplitudes are explicitly computed by (12). Rigid body motion modes $\left(\omega_{i}=0\right)$ are discarded and the nodal displacement are recovered using (8).

External forces $f_{i}$ used in (13) denote the $x$ and $y$ components of the forces acting on node $i$

$$
\mathbf{f}=\left[f_{x, 1}, f_{y, 1}, f_{x, 2}, f_{y, 2}, \ldots, f_{x, N}, f_{y, N}\right]^{T}
$$

where $N$ is the number of model nodes. Each force value is computed based on a distance transform metric between the model position and the object contour. A distance transform can be used to this end [20]-[22]. A distance transformation is an operation that converts a binary picture, consisting of contour points and background, to a grayscale picture where each pixel has an intensity proportional to its distance to the nearest contour point [23]. This distance transformation drives the external force generator

$$
f_{i}=\frac{1}{2} w\|d i s t\|^{2} .
$$

\section{Node CORRESPONDENCES AT DifFERENT SLICES}

Having modeled the object contours using the physics-based deformable model (14), the next step is to determine interslice correspondences between the contour model parameters, in 


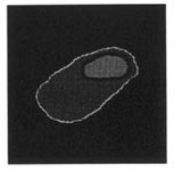

(a)

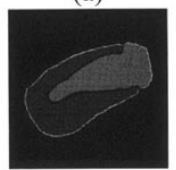

(e)

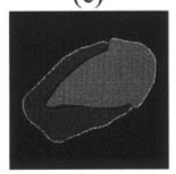

(i)

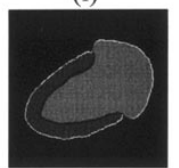

(m)

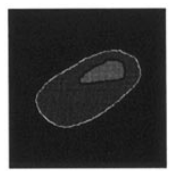

(b)

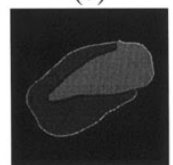

(f)

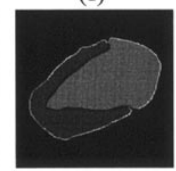

(j)

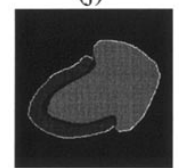

(n)

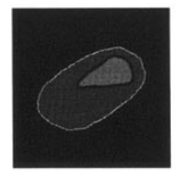

(c)

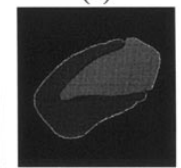

(g)

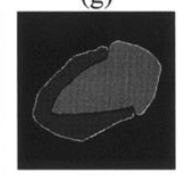

(k)

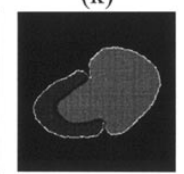

(o)

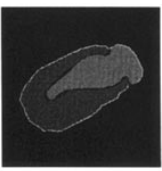

(d)

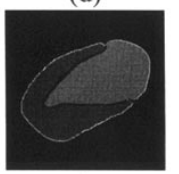

(h)

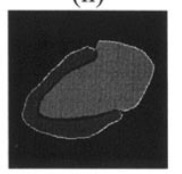

(1)

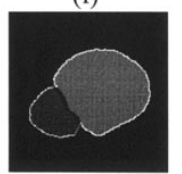

(p)
Fig. 2. Contour modeling of 2-D slices of a 3-D tooth germ volume of 265 slices. The deformable model at equilibrium is illustrated on 16 representative slices.

other words, the correspondences between the contour nodes of two slices.

To determine correspondences, we use vector $\mathbf{u}$ of (8), expressed as

$$
\mathbf{u}=\boldsymbol{\Phi} \tilde{\mathbf{u}}=\left[u_{x, 1}, u_{y, 1}, u_{x, 2}, u_{y, 2}, \ldots, u_{x, N}, u_{y, N}\right]^{T}
$$

where $N^{\prime}$ denotes the number of retained modes and $\left(u_{x, i}, u_{y, i}\right)$ describes the displacement of the $i$ th node at $X$ and $Y$ axis, respectively.

Thus, in order to determine the correspondences between two slices $Q$ and $S$, we build the generalized displacement vectors $\mathbf{u}^{Q}$ and $\mathbf{u}^{S}$, respectively, and compare them. The comparison of these vectors is based on a metric measuring the affinity between vector elements (contour nodes), i.e., a matrix which indicates how an element of the vector $\mathbf{u}^{Q}$ is related to the elements of the vector $\mathbf{u}^{S}$ (Section III-A). This node relation directly determines the node correspondences. Sections III-B and III-C describe how outlying and fault correspondences are rejected. Section III-D describes the computation of the desired node rotation and translation matrices.

\section{A. Affinity Matrix}

We now compute what are referred to as the affinities between the two generalized displacement vectors $\mathbf{u}^{Q}, \mathbf{u}^{S}$ (17). These are stored in a $N \times N$ affinity matrix $\mathbf{Z}^{Q S}$, where

$$
z_{i j}^{Q S}=\left\|x_{i}^{Q}-x_{j}^{S}\right\|^{2}+\left\|y_{i}^{Q}-y_{j}^{S}\right\|^{2} .
$$

$\|\cdot\|^{2}$ denotes the $L_{2}$ norm and $Q$ and $S$ are the slices under examination. The affinity measure $z_{i j}^{Q S}$ for the $i$ th and $j$ th nodes (of slices $Q$ and $S$, respectively), is zero for a perfect match and increases as the match worsens. Using the affinity metric, we can easily identify which node corresponds to each other in the two slices by looking for the minimum entry $z_{i j}^{Q S}$ in each column $j$ which is also the minimum in the corresponding row $i$ of matrix $\mathbf{Z}^{Q S}$. Because of the reduced basis matching, similarity of the generalized features is required in both directions (rows and columns) instead of one direction only (either row or column). In other words, a match between the $i$ th node in a certain slice and the $j$ th node in a candidate slice can only be valid if $z_{i j}^{Q S}$ is the minimum value for its row, and $z_{j i}^{Q S}$ the minimum for its column.

\section{B. Outlying Correspondence Rejection}

A measure $d^{Q S}$ expressing the continuity of correspondences between nodes (belonging to slices $Q$ and $S$ ), is also computed

$$
d^{Q S}\left(\boldsymbol{p}_{i}^{Q}\right)=\mid \mathcal{R} a n k\left(\boldsymbol{p}_{i}^{Q}\right)-\mathcal{R} \text { ank }\left(\Theta^{S}\left(p_{i}^{Q}\right)\right) \mid
$$

where $\boldsymbol{p}_{i}^{Q}=\left(x_{i}^{Q}, y_{i}^{Q}\right)$ denotes the $i$ th node of slice $Q, \Theta^{S}\left(\boldsymbol{p}_{i}^{Q}\right)$ its corresponding node on slice $S$ and $|\cdot|$ the $L_{1}$ norm. $\mathcal{R}$ ank $(\cdot)$ is a function measuring the position of a node in a contour with respect to a reference (starting) node [24]. Having computed $d^{Q S}\left(\boldsymbol{p}_{i}^{Q}\right)(19)$ for all node correspondences between slices $Q$ and $S$, our method accepts only correspondences satisfying [25], [26]

$$
d^{Q S}\left(\boldsymbol{p}_{i}^{Q}\right) \leq 1.4826 * \underset{k}{\operatorname{median}}\left\{d^{Q S}\left(\boldsymbol{p}_{k}^{Q}\right)\right\}
$$

where 1.4826 is the standard robust regression constant [25], [27]. Assuming that the continuity of correspondences $\boldsymbol{d}^{Q S}$ has a Gaussian distribution, the aforementioned constant is a robust threshold eliminating the correspondence outliers [25], [27]. These outliers are either anatomical structures appearing in one slice without a counterpart in other volume slices, or erroneous contour cuts created by tissue tears. In both cases, the condition (20) discards the outliers, enabling only the use of node correspondences with reasonable continuity.

An example of the previously described outlier rejection procedure is presented in Fig. 3. Two successive slices from a 3-D tooth germ volume are presented. For convenience, only five correspondences are selected. The nodes $\boldsymbol{p}_{1}^{i} \cdots \boldsymbol{p}_{5}^{i}$ of slice $i$ correspond to nodes $\boldsymbol{p}_{1}^{i+1} \cdots \boldsymbol{p}_{5}^{i+1}$ of slice $i+1$ according to (18). Their continuity measures are shown in Table I. As can be observed most of the correspondences have similar values, around 40 , apart from the last node correspondence $\left(p_{5}^{i}, p_{5}^{i+1}\right)$ having continuity 221 . This result can be extracted visually from Fig. 3, as the first four correspondences connect consecutive points, while the last one corresponds to an outlying node correspondence.

\section{Global Affinities}

Let us define a set of functions of type

$$
\mathcal{G}^{|Q-S|}\left(p_{i}^{Q}, p_{j}^{S}\right)= \begin{cases}1, & \text { if node } p_{i}^{Q} \text { corresponds to } p_{j}^{S} \\ 0, & \text { if there is no correspondence }\end{cases}
$$

where $p_{i}^{Q}$ is the $i$ th node of slice $Q$ and $p_{j}^{S}$ is the $j$ th node of slice $S$. Let us also define the correspondence matrix $\mathcal{C}_{Q, S}^{|Q-S|}$ between slices $Q$ and $S$ as follows:

$$
\mathcal{C}_{Q, S}^{|Q-S|}(i, j)=\left[\mathcal{G}^{|Q-S|}\left(p_{i}^{Q}, p_{j}^{S}\right)\right]
$$




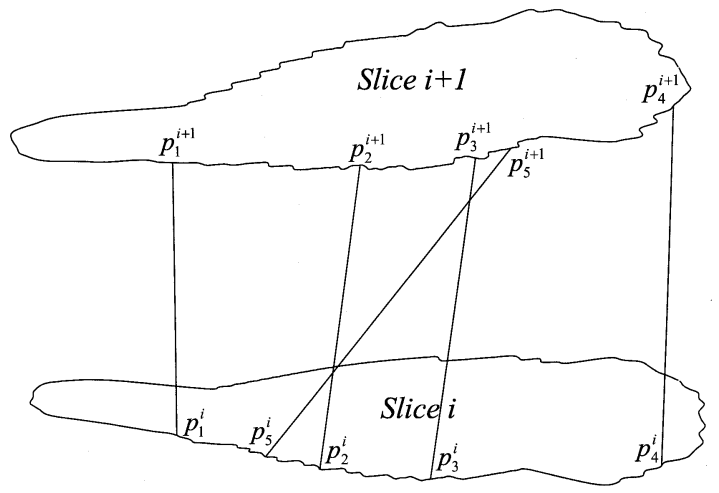

Fig. 3. Example of correspondences that minimize the contour affinity metric between points of two successive slices of a 3-D tooth germ. Only five correspondences are presented for convenience. The pair $\left(p_{5}^{i}, p_{5}^{i+1}\right)$ is a wrong correspondence.

TABLE I

OUTLYING CORRESPONDENCE REJECTION EXAMPLE. CONTINUITY MEASURES Between Five Points of Two Successive Slices (ShOwn IN Fig. 3)

\begin{tabular}{c|c|c|c|c|c}
\hline Slice $_{i}$ & Slice $_{i+1}$ & $\mathcal{R a n k}\left(p^{i}\right)$ & $\mathcal{R a n k}\left(p^{i+1}\right)$ & $d^{i, i+1}$ & decide \\
\hline \hline$p_{1}^{i}$ & $p_{1}^{i+1}$ & 10 & 49 & 39 & accept \\
\hline$p_{2}^{i}$ & $p_{2}^{i+1}$ & 51 & 93 & 42 & accept \\
\hline$p_{3}^{i}$ & $p_{3}^{i+1}$ & 163 & 204 & 41 & accept \\
\hline$p_{4}^{i}$ & $p_{4}^{i+1}$ & 257 & 295 & 38 & accept \\
\hline$p_{5}^{i}$ & $p_{5}^{i+1}$ & 18 & 239 & 221 & reject \\
\hline
\end{tabular}

where $\mathcal{C}_{Q, S}^{|Q-S|}$ is a $N \times N$ matrix, where $N$ is the total number of the contour nodes. Finally, we define the set of correspondence matrices between equidistant slices

$$
\mathcal{E}^{n}=\left\{\mathcal{C}_{1, n+1}^{n}, \mathcal{C}_{2, n+2}^{n}, \cdots, \mathcal{C}_{\Lambda-n, \Lambda}^{n}\right\}
$$

where $\Lambda$ is the total number of slices and $n$ is the distance between the slices under examination. If $n$ is equal to 1 , point correspondences are restricted to successive slice pairs.

Global affinities exploit and combine information from all node correspondence matrix sets, successive pairs $\mathcal{E}^{1}$, interlaced $\mathcal{E}^{2}$, etc. They can be used to discard any remaining fault correspondences as follows. Let us assume that we have the correspondence matrix sets $\mathcal{E}^{1}$ and $\mathcal{E}^{2}$ shown in Fig. 4. A correspondence between node $p_{i}^{n}$ of the $n$th slice and $p_{j}^{n+1}$ of slice $n+1$ is valid only if

$$
\mathcal{G}^{1}\left(p_{i}^{n}, p_{j}^{n+1}\right) \cdot \mathcal{G}^{1}\left(p_{i}^{n}, p_{k}^{n-1}\right) \cdot \mathcal{G}^{2}\left(p_{k}^{n-1}, p_{j}^{n+1}\right)=1
$$

where $k$ is a node belonging to the $(n-1)$ th slice. If expression (24) is verified, the initial correspondence between points $p_{i}^{n}$ and $p_{j}^{n+1}$ is accepted, otherwise it is discarded.

In other words, a correspondence between two slices is valid, only if it is sustained on more that one consecutive slices. For example, a correspondence $\left(p_{1}^{n+1}, p_{1}^{n}\right)$ from the $\mathcal{E}^{1}$ correspondence set is valid only if there is a valid correspondence $\left(p_{1}^{n}\right.$, $\left.p_{1}^{n-1}\right)$ and a correspondence $\left(p_{1}^{n+1}, p_{1}^{n-1}\right)$ in the $\mathcal{E}^{2}$ point correspondence set (Fig. 4). If even one of the aforementioned correspondences is missing, the correspondence $\left(p_{1}^{n+1}, p_{1}^{n}\right)$ is flagged as invalid. On the other hand, the correspondence $\left(p_{2}^{n+1}, p_{3}^{n-1}\right)$ is missing and consequently the correspondence $\left(p_{2}^{n+1}, p_{2}^{n}\right)$ is not accepted (the same stands for the nodes $p_{3}^{n+1}, p_{3}^{n}$ and $p_{4}^{n-1}$ ).

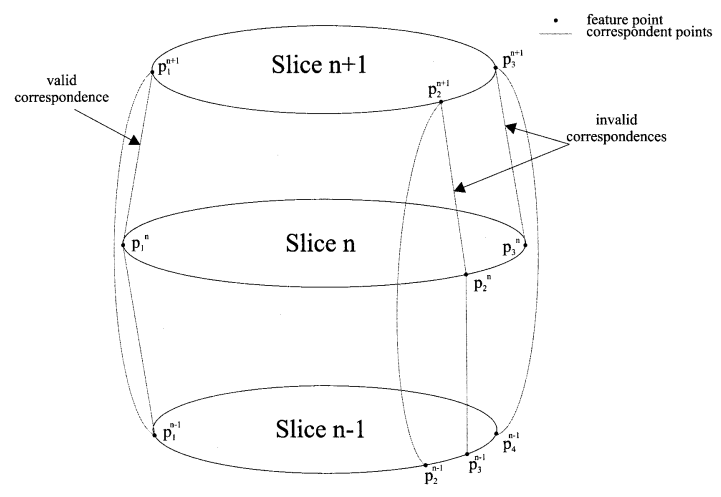

Fig. 4. Example of point correspondences between three successive slices. The points $\left[p_{1}^{n-1}, p_{1}^{n}, p_{1}^{n+1}\right]$ are considered as corresponding points, because the correspondences $\left[p_{1}^{n}, p_{1}^{n+1}\right],\left[p_{1}^{n-1}, p_{1}^{n}\right]$ and $\left[p_{1}^{n-1}, p_{1}^{n+1}\right]$ are all valid.

\section{Rotation and Translation Estimation}

After having validated and pruned the node correspondences, our algorithm determines the rigid transformation parameters (2-D rotation and translation) aligning the respective slices. The rotation matrix $\mathbf{R}$ and the translation vector $\mathbf{T}$ are estimated for each slice, in order to minimize the mean square error between the remaining corresponding nodes [28].

Let a sample covariance matrix $(2 \times 2)$ of the position of corresponding nodes $\boldsymbol{p}_{i}^{Q}$ and $\boldsymbol{p}_{i}^{S}$ in slices $Q$ and $S$, respectively, be

$$
\boldsymbol{C}_{Q S}=\frac{1}{L} \sum_{i=1}^{L}\left(\boldsymbol{p}_{i}^{Q}-\boldsymbol{\mu}_{Q}\right)\left(\boldsymbol{p}_{i}^{S}-\boldsymbol{\mu}_{S}\right)^{T}
$$

where $L$ is the number of corresponding nodes and $\boldsymbol{\mu}_{Q}$ and $\boldsymbol{\mu}_{S}$ are the mean vectors of the coordinates of the corresponding nodes in slices $Q$ and $S$, respectively. Let us perform a singular value decomposition of $\mathbf{C}_{Q S}$

$$
\boldsymbol{C}_{Q S}=\boldsymbol{U} \boldsymbol{D} \boldsymbol{V}^{T}, \quad \boldsymbol{D}=\operatorname{diag}\left(d_{i}\right), d_{1} \geq d_{2} \geq 0 .
$$

When $\operatorname{rank}\left(\boldsymbol{C}_{Q S}\right) \geq 1$, the optimum rigid object transformation parameters are determined uniquely as follows [28]:

$$
\begin{aligned}
\boldsymbol{R} & =\boldsymbol{U} \boldsymbol{P} \boldsymbol{V}^{T} \\
\boldsymbol{T} & =\boldsymbol{\mu}_{Q}-c \boldsymbol{R} \boldsymbol{\mu}_{S} \\
c & =\frac{1}{\sigma_{S}^{2}} \operatorname{tr}(\boldsymbol{D P}) \\
\sigma_{S}^{2} & =\frac{1}{L} \sum_{i=1}^{L}\left\|\boldsymbol{p}_{i}^{S}-\boldsymbol{\mu}_{S}\right\|^{2}
\end{aligned}
$$

and

$$
\mathbf{P}=\left\{\begin{array}{ll}
\mathbf{I} & \text { if } \operatorname{det}\left(\mathbf{C}_{Q S}\right) \geq 0 \\
\operatorname{diag}(1,-1) & \text { if } \operatorname{det}\left(\mathbf{C}_{Q S}\right)<0
\end{array} .\right.
$$

Finally, the overall alignment algorithm is summarized in Fig. 5.

\section{EXPERIMENTAL RESULTS}

To evaluate our method, we applied the proposed algorithm to the reconstruction of a large number of volumes. We used volumes (e.g., Figs. 6 and 7) with known ground truth, i.e., aligned 
- for each slice do

- deform the initial circular physics-based model (11-14)

- end for

- for each successive slice pairs do

- compute correspondence affinities (18), i.e. compute nodal correspondences

- eliminate outlying correspondences (19-20)

- end for

- determine the correspondence sets $\left(\mathcal{E}^{1}, \mathcal{E}^{2}\right.$, etc), (23)

- for each slice $n$ do

- for each node $i$ do

* if node $i$ has correspondence in $\mathcal{E}^{1}$ (correspondent point $j$ in slice $n+1$ do

- compute expression (24)

- if (24) is equal to zero, discard the correspondence

- else, accept the correspondence

- end if

$*$ end if

- end for

- end for

- for each slice do

- estimate rotation and translation

- apply transformations

- end for

Fig. 5. Flowchart of the overall alignment algorithm.

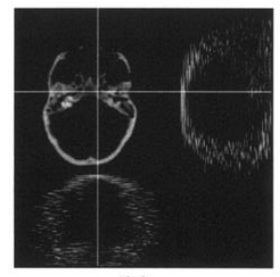

(a)

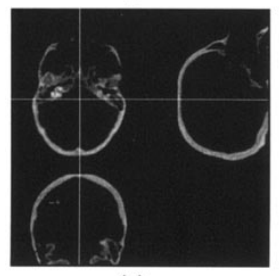

(c)

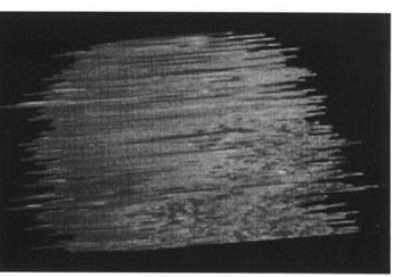

(b)

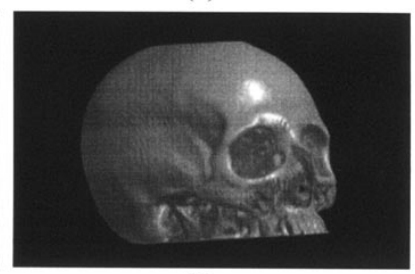

(d)

Fig. 6. Reconstruction of a 3-D human skull volume of 140 slices (a) Multiplanar view of the volume before registration. (b) 3-D view of the volume before registration. (c) Multiplanar view of the volume after registration. (d) 3-D view of the volume after registration.

volumes which are used as reference for the performance of the algorithm. They are manually, artificially misaligned, i.e. they are randomly transformed using translations $\left(t_{x}, t_{y}\right)$ with $t_{x}, t_{y}$ varying from -10 to +10 pixels and rotations $\theta$ varying from -40 to +40 degrees, in order to measure and confirm the registration accuracy and efficiency of our algorithm. Moreover, we applied our method on volumes with unknown ground truth (e.g., Figs. 8-11), i.e., volumes acquired by a microtome, with no reference point. Thus, the alignment of these volumes is completely blind, and an efficient way to evaluate the results is a manual evaluation by the physician-researcher who originally provided the volumes and is aware of their appearance.
Furthermore, in order for our algorithm to operate properly, the existence of an obvious foreground and background in the volumes, is necessary, i.e., the object under alignment needs to be segmented, either manually or automatically [29].

As aforementioned, our method was applied to the reconstruction of an artificially misaligned 3-D human skull shown in Fig. 6. Our method is not intended to be used for CT or MRI images, since they are inherently aligned. We present the CT examples only to show that we can recover the 3-D volumes after artificial misalignment and verify the performance of our method. Thus, the slices of the original $256 \times 256 \times 140 \mathrm{CT}$ volume were transformed using translations $\left(t_{x}, t_{y}\right)$ with $t_{x}$, $t_{y}$ varying from -10 to +10 pixels and rotations $\theta$ varying from -40 to +40 degrees. The transformation parameters of translation and rotation for each slice followed a uniform distribution. The misaligned slices are shown in Fig. 6(a) and (b). The human skull volume has discontinuities and consecutive slices may differ significantly due to skull anatomy. However, the correspondence evaluation was proven robust to these shortcomings. Table II presents statistics on the alignment parameter errors $\Delta t_{x}, \Delta t_{y}$ and $\Delta \theta$. As can be seen, median and mean translation and rotation errors are less than 1 pixel and 1 degree, respectively. Also, maximum errors are slightly larger than 1 pixel and 1 degree, respectively, showing the robustness of the proposed technique. Fig. 6(c) and (d) present the realigned volume.

The same evaluation procedure was performed on a 3-D CT scanned volume of a mechanical part with 109 slices shown in Fig. 7. The algorithm aligned the artificially (randomly) misaligned slices of the volume. The alignment parameter errors are presented in Table III. As can be seen, median and mean translation and rotation errors are less than 1 pixel and 1 degree, respectively. Also maximum errors are slightly larger than 1 pixel 


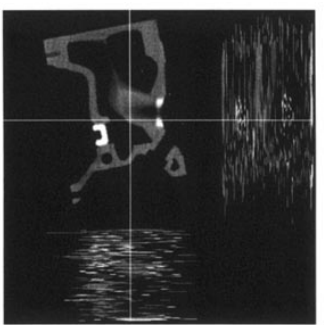

(a)

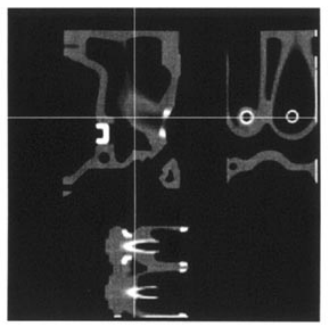

(c)

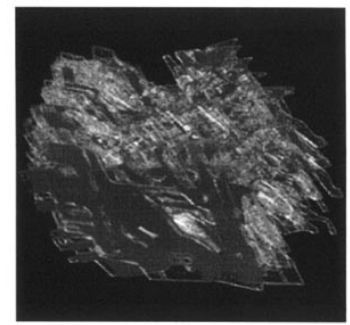

(b)

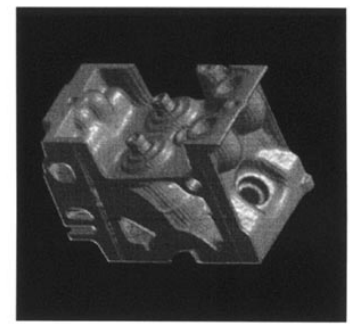

(d)
Fig. 7. Reconstruction of a 3-D scanned mechanical part volume of 109 slices. (a) Multiplanar view of the volume before registration. (b) 3-D view of the volume before registration. (c) Multiplanar view of the volume after registration. (d) 3-D view of the same volume after registration.

TABLE II

Alignment PARAmeter ERror Statistics of A SET of A 3-D CT HuMAN SkUll Volume ReAlignment By the Proposed Method (Shown IN Fig. 6)

\begin{tabular}{l|c|c|c}
\hline & $\Delta t_{x}$ & $\Delta t_{y}$ & $\Delta \theta$ \\
\hline \hline median & 0.19 & 0.23 & 0.13 \\
\hline maximum & 1.18 & 1.07 & 1.42 \\
\hline mean \pm s. dev & $0.29 \pm 0.26$ & $0.31 \pm 0.26$ & $0.38 \pm 0.57$ \\
\hline
\end{tabular}

and 1 degree, respectively, showing the robustness of the proposed technique.

Furthermore, the algorithm was applied to the reconstruction of tooth germ volumes with unknown ground truth. The performance of our method was compared to the manual alignment accomplished by an expert physician-researcher. Fig. 8 shows the reconstruction of a tooth germ by an expert dentist-researcher [Fig. 8(a) and (b)] and by our method [Fig. 8(c) and (d)]. It is illustrated that human intervention fails to correctly align the slices, whilst our method produces much faster alignment with higher accuracy, as confirmed by dentist specialists-researchers. The same stands for the examples presented in Figs. 9-11, where the reconstruction of other three teeth volumes is presented. In all the examples, a specialist (dentist-researcher) confirmed that the volumes reconstructed by our method are of higher accuracy and visual quality. One should note that the specialist needs approximately $2-3 \mathrm{~d}$ to manually align a volume, whereas our algorithm performs the reconstruction of the same volume in a minute, with better results as confirmed by the specialist. Additionally, one can take into consideration that the specialist is the only person who knows the object under registration before its digitization and its real appearance. This can also be observed by a simple visual inspection (Figs. 8-11), especially at their multiplanar view.

Sometimes, during serial sectioning, some slices tend to be very deformed and have variable slice thickness. Thus, we

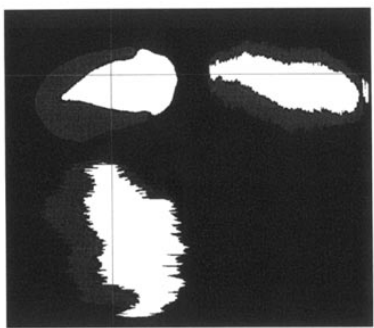

(a)

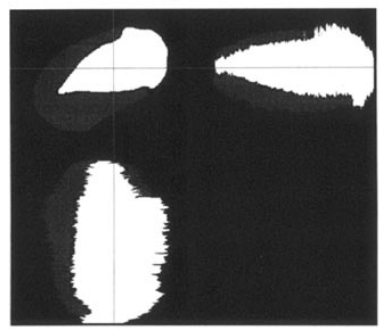

(c)

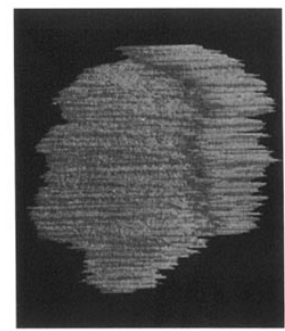

(b)

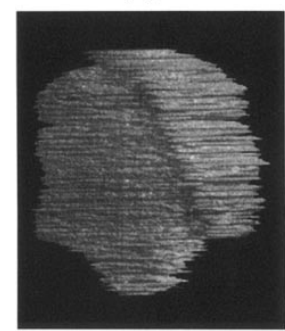

(d)
Fig. 8. Reconstruction of a 3-D tooth germ volume of 265 slices. (a) Multiplanar and (b) 3-D view of the volume after manual alignment by an expert dentist. (c) Multiplanar and (d) 3-D view of the volume after automatic alignemnt.

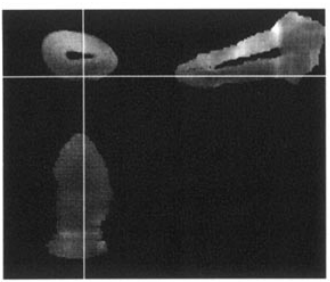

(a)

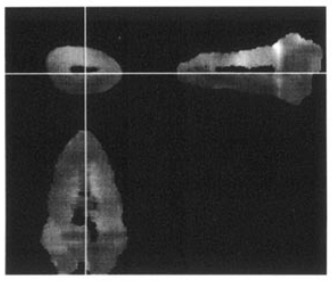

(c)

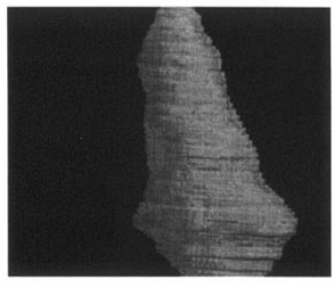

(b)

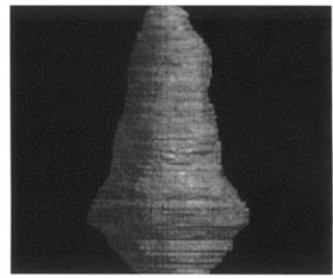

(d)
Fig. 9. Reconstruction of a 3-D tooth germ volume of 194 slices. (a) Multiplanar. (b) 3-D view of the volume after manual alignment by an expert dentist. (c) Multiplanar. (d) 3-D view of the volume after registration.

TABLE III

AlignMENT PARAMETER ERror Statistics of a SET OF A 3-D CT SCANNED MECHANICAL PART VOLUME REALIGNMENT BY THE Proposed METHOd (ShOWN IN FIG. 7)

\begin{tabular}{l|c|c|c}
\hline & $\Delta t_{x}$ & $\Delta t_{y}$ & $\Delta \theta$ \\
\hline \hline median & 0.33 & 0.31 & 0.17 \\
\hline maximum & 1.07 & 0.93 & 1.75 \\
\hline mean \pm s. dev & $0.38 \pm 0.36$ & $0.42 \pm 0.39$ & $0.35 \pm 0.41$ \\
\hline
\end{tabular}

applied our algorithm to the reconstruction of some artificially misaligned and volumes having varying slice thickness (3-D human skull and 3-D CT scanned mechanical part). The artificial misalignment was random and performed as described previously, and the slice thickness variation was achieved using two different and randomly chosen sets of sequential slices 


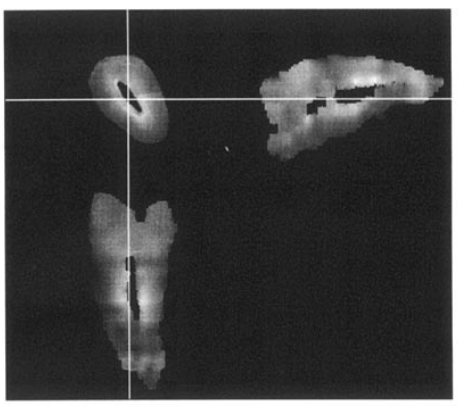

(a)

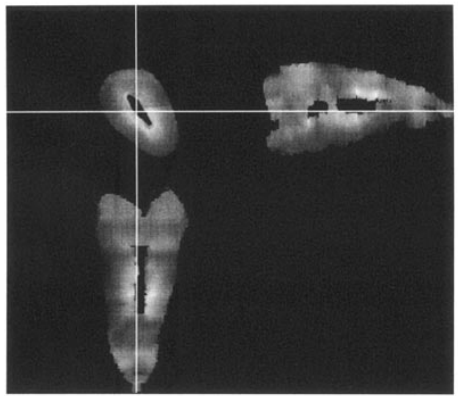

(c)

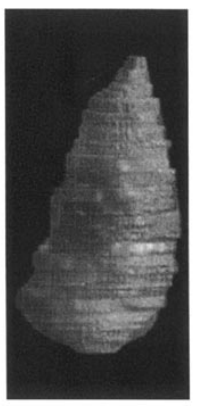

(b)

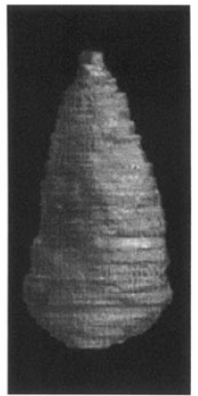

(d)
Fig. 10. Reconstruction of a 3-D tooth germ volume of 169 slices. (a) Multiplanar. (b) 3-D view of the volume after manual alignment by an expert dentist. (c) Multiplanar. (d) 3-D view of the volume after registration.

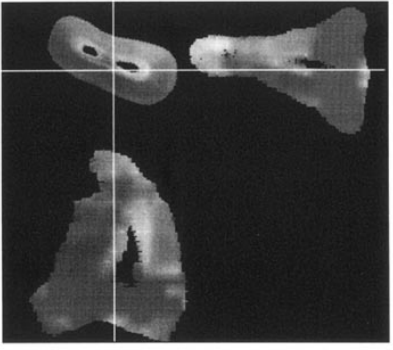

(a)

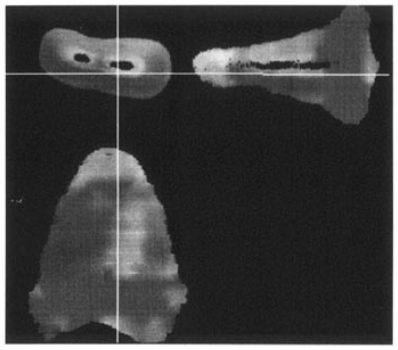

(c)

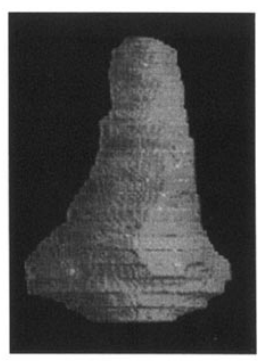

(b)

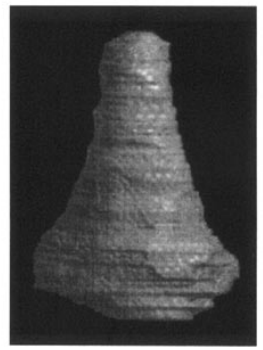

(d)
Fig. 11. Reconstruction of a 3-D tooth germ volume of 161 slices. (a) Multiplanar. (b) 3-D view of the volume after manual alignment by an expert dentist. (c) Multiplanar. (d) 3-D view of the volume after registration.

(ten slices each) from both volumes and fulfilled as depicted in Figs. 12 and 13. Tables IV and V present the alignment errors of our method. Although the errors are higher than the corresponding ones, when there is no difference in slice thickness, they are still very low and satisfactory, showing the robustness of the proposed technique.

Moreover, we applied the algorithm to the realignment of an original already aligned 3-D human skull volume (the original volume used in Fig. 6). The results shown in Table VI illustrate

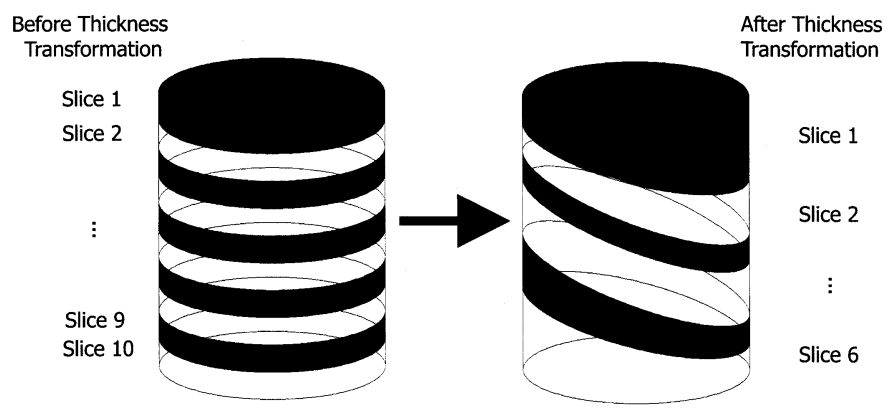

Fig. 12. Slice thickness transformation in ten slices of a volume. 10 parallel slices are transformed in order to have variable thickness.

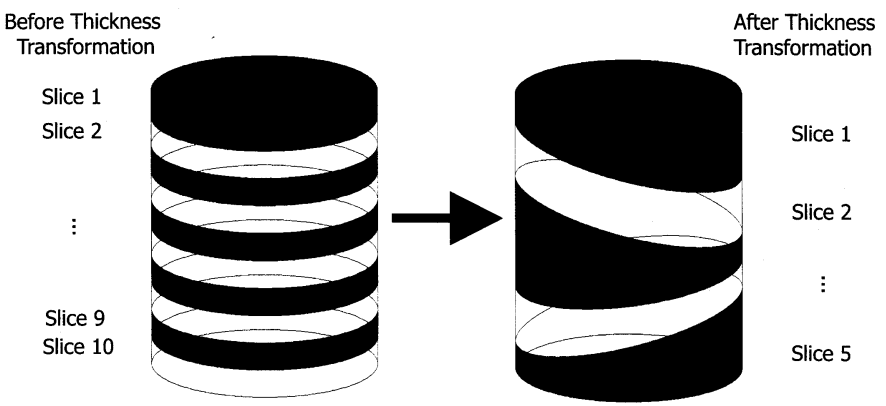

Fig. 13. Slice thickness transformation in ten slices of a volume. 10 parallel slices are transformed in order to have variable thickness.

TABLE IV

Alignment ERror Statistics of a Set of A 3-D CT Human SKUll Volume With Slice Thickness Variation (ShOWn IN Figs. 12 AND 13) REALIGNED BY THE PROPOSED METHOD

\begin{tabular}{l|c|c|c}
\hline & $\Delta t_{x}$ & $\Delta t_{y}$ & $\Delta \theta$ \\
\hline \hline median & 0.22 & 0.34 & 0.15 \\
\hline maximum & 1.58 & 1.67 & 2.42 \\
\hline mean $\pm \mathrm{s} . \mathrm{dev}$ & $0.43 \pm 0.47$ & $0.41 \pm 0.51$ & $0.42 \pm 0.62$ \\
\hline
\end{tabular}

TABLE V

AlignMENT ERror Statistics of a SET of A 3-D CT SCANNED Mechanical Part Volume With Slice Thickness Variation (Shown in Figs. 12 And 13) Realigned by the Proposed Method

\begin{tabular}{l|c|c|c}
\hline & $\Delta t_{x}$ & $\Delta t_{y}$ & $\Delta \theta$ \\
\hline \hline median & 0.32 & 0.39 & 0.20 \\
\hline maximum & 1.79 & 1.91 & 2.05 \\
\hline mean \pm s. dev & $0.48 \pm 0.52$ & $0.51 \pm 0.59$ & $0.37 \pm 0.42$ \\
\hline
\end{tabular}

that our algorithm is unbiased. As can be seen, median and mean translation and rotation errors are less that 0.2 pixel and 0.1 degree, respectively. Also, maximum errors are about 1 pixel and less than 0.5 degree, respectively, proving the unbiasedness of our method.

Finally, let us notice that the algorithm has a computational complexity $O(N M)$, where $N$ is the number of slices and $M$ is the number of nodes of the deformable model. The computation time is approximately $1 \mathrm{~min}$ for the reconstruction of a $256 \times$ $256 \times 140$ volume on a Pentium III $(700 \mathrm{MHz})$ workstation under Windows 2000 Professional without any particular code optimization. 
TABLE VI

ALIGNMENT ERror Statistics OF an ALREAdy ALigned SET OF A 3-D CT Human SKUll Volume (THE GROUND TRUth) That waS APPLIED TO THE PROPOSED ALGORITHM

\begin{tabular}{l|c|c|c}
\hline & $\Delta t_{x}$ & $\Delta t_{y}$ & $\Delta \theta$ \\
\hline \hline median & 0.17 & 0.21 & 0.05 \\
\hline maximum & 0.98 & 1.03 & 0.89 \\
\hline mean $\pm \mathrm{s} . \mathrm{dev}$ & $0.22 \pm 0.23$ & $0.27 \pm 0.24$ & $0.33 \pm 0.48$ \\
\hline
\end{tabular}

\section{CONCLUSION}

A fast and robust algorithm for the alignment of 2-D serially acquired slices was presented. A 2-D physics-based deformable contour modeling technique was used to parameterized the contour of each slice of the volume under registration. The model parameters was used to establish mode correspondences among contour nodes of consecutive slices using special mode affinity metrics. Local and global constraints was exploited to prune node correspondences that do not make sense, and finally, various slices were aligned by using valid node correspondences and the calculation of the optimal rotation and translation matrices. The proposed technique is proven to produce very low alignment errors. It is much faster and more accurate than manual alignment.

Furthermore, no particular registration direction is privileged by the proposed approach and the use of a global affinity measure eliminates error propagation. Also, the low frequency modal parameterization of the object contours makes the technique robust to missing data or outliers and to slice thickness variability.

The low computation time and the good quality of the alignment with respect to manual techniques makes the method a promising tool for the reconstruction of 3-D anatomical structures.

\section{ACKNOWLEDGMENT}

The authors wish to thank Professor K. Lyroudia, Department of Dentistry, Aristotle University of Thessaloniki, Greece, and A. Digka, dentist and Ph.D. degree candidate in endodontology, Department of Dentistry, Aristotle University of Thessaloniki, for providing the dental images and evaluating the registration results.

\section{REFERENCES}

[1] L. Gottesfeld-Brown, "A survey of image registration techniques," $A C M$ Computing Surveys, vol. 24, no. 4, pp. 325-376, 1992.

[2] J. B. A. Maintz and M. A. Viergever, "A survey of medical image registration techniques," Medical Image Analysis, vol. 2, no. 1, pp. 1-36, 1998.

[3] P. V. den Elsen, E. J. D. Paul, and M. A. Viergever, "Medical image matching-a review with classification," IEEE Eng. Med. Biol., vol. 12, pp. 26-39, 1993.

[4] A. F. Goldszal, O. J. Tretiak, P. J. Hand, S. Bhasin, and D. L. M. Eachron, "Three-dimensional reconstruction of activated columns from $2-\left[{ }^{14} \mathrm{C}\right]$ Deoxy-D-glucose data," NeuroImage, vol. 2, pp. 9-20, 1995.
[5] E. Bardinet, L. D. Cohen, and N. Ayache, "Analyzing the deformation of the left ventricle of the heart with a parametric deformable model," INRIA Sophia Antipolis, France, Tech. Rep. RR-2797, 1996.

[6] L. Hibbard and R. Hawkins, "Objective image alignment for three-dimensional reconstruction of digital autoradiographs," J. Neurosci. Meth., vol. 26, pp. 55-75, 1988.

[7] A. Rangarajan, H. Chui, E. Mjolsness, S. Pappu, L. Davachi, P. Goldman-Rakic, and J. Duncan, "A robust point-matching algorithm for autoradiograph alignment," Medical Image Analysis, vol. 1, no. 4, pp. 379-398, 1997.

[8] W. Zhao, T. Young, and M. Ginsberg, "Registration and three-dimensional reconstruction of autoradiographic images by the disparity analysis method," IEEE Trans. Med. Imaging, vol. 12, no. 4, pp. 782-791, 1993.

[9] A. Andreasen, A. M. Drewes, J. Assentoft, and N. E. Larsen, "Computer-assisted alignment of standard serial sections without use of artificial landmarks. A practical approach to the utilization of incomplete information of 3-D reconstruction of the hippocampal region," J. Neurosci. Meth., vol. 45, pp. 199-207, 1992.

[10] B. Kim, J. Boes, K. Frey, and C. Meyer, "Mutual information for automated unwarping of rat brain autoradiographs," NeuroImage, vol. 5, pp. 31-40, 1997.

[11] S. Ourselin, A. Roche, G. Subsol, X. Pennec, and C. Sattonnet, "Automatic alignment of histological sections for 3-D reconstruction and analysis," INRIA, Sophia Antipolis, France, Tech. Rep. 3595, 1998.

[12] C. Nastar and N. Ayache, "Frequency-based nonrigid motion analysis: application to four dimensional medical images," IEEE Trans. Pattern Anal. Machine Intell., vol. 18, pp. 1069-1079, 1996.

[13] C. Nikou, G. Bueno, F. Heitz, and J. P. Armspach, "A joint physicsbased statistical deformable model for multimodal brain image analysis," IEEE Trans. Med. Imag., vol. 20, pp. 1026-1037, 2001.

[14] S. Sclaroff and A. Pentland, "Modal matching for correspondence and recognition," IEEE Trans. Pattern Anal. Machine Intell., vol. 17, pp. 545-561, June 1995.

[15] A. Pentland and S. Sclaroff, "Closed-form solutions for physically-based shape modeling and recognition," IEEE Trans. Pattern Anal. Machine Intell., vol. 13, pp. 730-742, 1991 .

[16] A. Pentland and B. Horowitz, "Recovery of nonrigid motion and structure," IEEE Trans. Pattern Anal. Machine Intell., vol. 13, pp. 730-742, July 1991.

[17] K. J. Bathe, Finite Element Procedure. Englewood Cliffs, NJ: Prentice-Hall, 1996.

[18] C. Nastar, "Modles physiques deformables et modes vibratoires pour l'analyze du mouvement nonrigide dans les images multidimensionnelles," Ph.D. dissertation, Ecole Nationale des Ponts et Chaussees, 1994.

[19] G. Borgefors, "On digital distance transforms in three dimensions," Computer Vision and Image Understanding, vol. 64, no. 3, pp. 368-376, 1996.

[20] P.-E. Danielsson, "Euclidean distance transform," Computer Graphics and Image Processing, vol. 14, pp. 227-228, 1980

[21] G. Borgefors, "Distance transformations in arbitrary dimensions," Computer Vision, Graphics, and Image Processing, vol. 27, pp. 321-345, 1984.

[22] J. Canny, "A computational approach to edge detection," IEEE Trans. Pattern Anal. Machine Intell., vol. 8, pp. 679-698, Nov. 1986.

[23] G. Borgefors, "Hierarchical chamfer matching: a parametric edge matching algorithm," IEEE Trans. Pattern Anal. Machine Intell., vol. 10, pp. 849-965, Nov. 1988.

[24] H. A. David, Order Statistics: Wiley \& Sons, 1981.

[25] P. J. Rousseeuw, "Least median of squares regression," J. Amer. Statistical Assoc., vol. 79, pp. 871-880, 1984.

[26] M. J. Black and A. Rangarajan, "On the unification of line processes, outlier rejection, and robust statistics with applications in early vision," Int. J. Computer Vision, vol. 19, no. 1, pp. 57-91, 1996.

[27] P. J. Rousseeuw and A. M. Leroy, Robust Regression and Outlier Detection: Wiley, 1987.

[28] S. Umeyama, "Least-squares estimation of transformation parameters between two point patterns," IEEE Trans. Pattern Anal. Machine Intell., vol. 13, pp. 376-380, Apr. 1991.

[29] A. G. Bors and I. Pitas, "Optical flow estimation and moving object segmentation based on median radial basis function network," IEEE Trans. Image Processing, vol. 7, pp. 693-702, May 1998. 


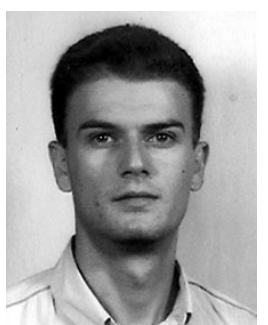

Stelios Krinidis was born in Kavala, Greece, in 1978. He received the Diploma in Informatics from the Aristotle University of Thessaloniki, Greece, in 1999.

In June 2000, he joined the Artificial Intelligence and Information Analysis Group at the same University, where he is currently a researcher and teaching assistant and is pursuing the Ph.D. degree on 3-D image processing. His research interests include 3-D image processing and analysis and computer vision.

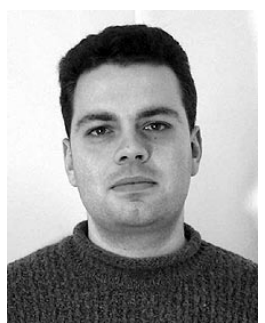

Christophoros Nikou (M'02) was born in Thessaloniki, Greece, in 1971. He received the diploma in electrical engineering from the Aristotle University of Thessaloniki, in 1994. He received the DEA in optoelectronics and image processing in 1995 and the $\mathrm{Ph} . \mathrm{D}$. degree in image processing and computer vision in 1999, both from Louis Pasteur University of Strasbourg, France.

During 2001, he was a Senior Researcher with the Department of Informatics, Aristotle University of Thessaloniki, where he conducted research in image processing in the framework of various European projects. Since 2002, he has been with Compucon S.A., Thessaloniki, managing research projects in 3-D medical image processing and analysis. His research interests mainly include computer vision, pattern recognition, biomedical image processing, image registration and segmentation, deformable models, and statistical image processing.

Dr. Nikou is a member of the Technical Chamber of Greece.

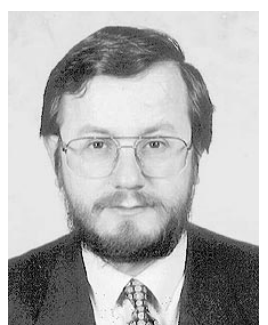

Ioannis Pitas (SM'94) received the Diploma of Electrical Engineering in 1980 and the Ph.D. degree in electrical engineering in 1985 , both from the University of Thessaloniki, Greece.

Since 1994, he has been a Professor with the Department of Informatics, University of Thessaloniki. From 1980 to 1993, he served as Scientific Assistant, Lecturer, Assistant Professor, and Associate Professor with the Department of Electrical and Computer Engineering at the same University. He served as a Visiting Research Associate with the University of Toronto, Canada, University of Erlangen-Nuernberg, Germany, Tampere University of Technology, Finland, as Visiting Assistant Professor with the University of Toronto and as Visiting Professor with the University of British Columbia, Vancouver, Canada. He was a Lecturer in short courses for continuing education. His current interests are in the areas of digital image processing, multidimensional signal processing, watermarking, and computer vision. He has published more than 380 papers and contributed in 13 books in his areas of interest. He is the coauthor of the books Nonlinear Digital Filters: Principles and Applications (Boston, MA: Kluwer, 1990), 3-D Image Processing Algorithms (New York: Wiley, 2000), Nonlinear Model-Based Image/Video Processing and Analysis (New York: Wiley, 2001), and is the author of Digital Image Processing Algorithms and Applications (New York: Wiley, 2000). He is the editor of the book Parallel Algorithms and Architectures for Digital Image Processing, Computer Vision and Neural Networks (New York: Wiley, 1993).

Dr. Pitas has been member of the European Community ESPRIT Parallel Action Committee. He has also been an invited speaker and/or member of the program committee of several scientific conferences and workshops. He was Associate Editor of the IEEE TRANSACTIONS ON CIRCUITS AND SySTEMS, Associate Editor of the IEEE TRANSACTIONS ON NEURAL NETWORKS, Coeditor of Multidimensional Systems and Signal Processing, and he is currently an Associate Editor of the IEEE TRANSACTIONS ON IMAGE PRocessing. He was General Chair of the 1995 IEEE Workshop on Nonlinear Signal and Image Processing (NSIP95), Technical Chair of the 1998 European Signal Processing Conference, and General Chair of IEEE ICIP2001. 